\title{
Actualización: Problemas en los pies en los pacientes con diabetes (primera entrega): generalidades y manejo de las úlceras
}

\author{
Feet problems in diabetic patients (first part): general approach and management of ulcers
}

Adriana Primerano*

\begin{abstract}
Resumen
La afección del pie en los pacientes con diabetes constituye por su importancia una entidad en si misma. En el espectro de su afectación encontraremos desde simples cambios en la piel, hasta lesiones necróticas y osteomielitis, que pueden conducir a amputaciones y aún, comprometer la vida del paciente. En la presente revisión se consideran aspectos generales del pie de las personas con diabetes y en particular, sobre el abordaje de las úlceras. En una entrega posterior se profundizará sobre el diagnóstico y manejo de las infecciones del pie en estos pacientes.
\end{abstract}

\section{Abstract}

Foot involvement in diabetic patients is an entity by itself. The spectrum of their affection range from skin changes to necrotic lesions and osteomyelitis, which can lead to amputations and even compromise the patient's life. In this review, general aspects of diabetic foot are considered addressing the ulcers in particular. In a subsequent issue, diagnosis and management of diabetic foot infections will be reviewed.

Palabras clave: pie diabético, úlceras, diagnóstico, tratamiento. Key words: diabetic foot ulcers, diagnosis, treatment.

Primerano A. Problemas en los pies de los pacientes con diabetes: generalidades y manejo de las úlceras. Evid. actual. práct. ambul; 11(3):83-86, MayJun.2008.

Introducción

La diabetes mellitus en general, y la de tipo 2 en particular, constituyen un importante problema de salud a nivel mundial y cuya prevalencia está en aumento, calculándose que para 2025 y comparándola con la de 1995, aumentaría un 35\%.

Se estima que la atención de los pacientes con diabetes genera el doble de gastos que la de los no afectados por esta condición clínica, en gran medida generados por la atención de sus complicaciones crónicas ${ }^{1,2}$. Entre estas, una de las más temidas es la afección del pie. Sin embargo y a pesar del impacto que esta entidad produce en la calidad de vida, de su costo socioeconómico, de su alta frecuencia de aparición y de su carácter de entidad prevenible, la evaluación de los pies no suele formar parte efectiva de los controles rutinarios del paciente con diabetes, lo que suele conducir que el diagnóstico y el tratamiento de sus complicaciones no se realice con la celeridad y pericia adecuados.

Aspectos epidemiológicos de los problemas de los pies en la población con diabetes

La afección de las extremidades inferiores en los pacientes con diabetes es dos veces más frecuente que en la población general y afecta aproximadamente a uno de cada tres pacientes mayores de $40 \mathrm{años}^{2}$. En una persona con diabetes, el riesgo de desarrollar una ulceración en el pie a lo largo de su vida ronda el $15 \%$, oscilando su incidencia anual entre 1 y $4 \%$, y su prevalencia entre 4 y $10 \% \%^{2,3}$. Las úlceras de pie diabético son la principal causa de ingreso hospitalario en los pacientes con diabetes, además de ser la complicación que insume mayor estadía de internación.

Estas afecciones suponen múltiples consecuencias, siendo la más temida la amputación. Las amputaciones del pie son 20 veces más frecuentes en los pacientes con diabetes que en la población general ${ }^{4}$, siendo precedidas por úlceras en el $85 \%$ de los casos $^{2}$. Los pacientes amputados tienen peor pronóstico. Por ejemplo, la incidencia de una nueva úlcera o de una amputación contralateral ronda el $50 \%$ y su sobrevida media a cinco años es del $40 \%{ }^{2}$.

\section{Fisiopatogenia}

El 45 al $60 \%$ de las úlceras serían de origen neuropático, el 10 al $15 \%$ de origen isquémico, y el 25 a $45 \%$ de origen mixto, neuropático e isquémico ${ }^{2}$; lo que hace que la neuropatía esté implicada en un 85 a $90 \%$ de los casos. Por lo tanto un pie insensible, esté bien prefundido o no es un pie de riesgo.

- Las alteraciones de las fibras sensitivas (neuropatía sensitiva) dan como resultado la disminución o pérdida de la protección del pie que supone la sensibilidad dolorosa.

- Las alteraciones en las fibras autonómicas (neuropatía autonómica) ocasionan una alteración de las funciones vasomotoras y del reflejo de sudoración del pie, cuya consecuencia directa son la disminución del flujo sanguíneo capilar -secundaria a la apertura de puentes arteriovenosos- y la sequedad cutánea. Estas a su vez condicionan la aparición de grietas, con la consecuente aparición de una puerta de entrada de infecciones ${ }^{5}$

- Las alteraciones de las fibras motoras (neuropatía motora) se asocian a debilidad y atrofia de los músculos intrínsecos del pie, lo cual provoca limitaciones en su movilidad. Aun no está aclarado si es éste el mecanismo por el cual aparecen las deformidades óseas propias del pie diabético (dedos en garra o en martillo $)^{6}$ que generan puntos anómalos de presión y lo hacen más vulnerable a pequeños traumatismos externos producto del diario caminar y/o el uso de calzado inapropiado $0^{5,7}$.

Sin embargo, otros mecanismos concurrentes convierten al pie de las personas con diabetes en vulnerable a las lesiones e infecciones, entre ellos:

- La macroangiopatía diabética, que evoluciona hacia la isquemia progresiva en los miembros inferiores ${ }^{5}$. Las lesiones ateromatosas suelen ser segmentarias, bilaterales y más prevalentes en los segmentos infrageniculares y distales.

- La microangiopatía diabética, que afecta a los capilares, arteriolas y vénulas de todo el organismo. Su importancia en la etiopatogenia de la úlcera del pie diabético parece ser menor de lo que se creía anteriormente. Por si misma, es poco probable que cause la úlcera, pero si está involucrada en la producción de la necrosis tisular ${ }^{5}$.

- Las alteraciones inmunológicas que participan una vez instaurada la úlcera, favoreciendo la aparición y la progresión de las infecciones ${ }^{2,5}$. Se han descrito múltiples defectos en la respuesta inmune del paciente diabético, con clara afección de la serie blanca en fenómenos como la diapédesis, adherencia, quimiotaxis y capacidad destructiva de los polimorfos nucleares; y defectos agravados en el contexto de malos controles de la glucemia $^{5}$.

La consecuencia final es la aparición de la úlcera, cuya secuen-

* Servicio de Medicina Familiar y Comunitaria. Hospital Italiano de Bs. As. adriana.primerano@ hospitalitaliano.org.a 
cia habitual de aparición se podría resumir del siguiente modo: el hecho inicial suele ser tan banal como una pequeña fisura producida en cualquier punto del pie. A partir de aquí penetran los gérmenes y producen una infección localizada, que pasa muchas veces inadvertida.

Desde aquí, la infección se extiende por las vainas tendinosas del pie conduciendo a necrosis tisular, pudiéndose observar la oclusión trombótica de los pequeños vasos sanguíneos. La isquemia resultante facilita la extensión de la infección y el crecimiento de gérmenes anaerobios. Esta "microangiopatia infectiva" puede destruir las barreras fibrosas (aponeurosis plantar, vainas tendinosas y otras estructuras) y la piel, permitiendo que la infección se propague a los extremos distales del pie. Aparecen entonces áreas de gangrena, que pueden ser secas o húmedas según la rapidez del proceso, y que determinarán la posibilidad de evolución hacia la amputación del miembro ${ }^{5}$.
Abordaje de los problemas en los pies de los pacientes con diabetes

La clave en el abordaje es la prevención. Si bien todos los pacientes deben ser educados sobre el autocuidado de sus pies, esto resulta de especial importancia en aquellos con alteración de la sensibilidad. Se deberá instruir al paciente sobre los cuidados del pie: hidratación de la piel, cuidado de las uñas y elección del calzado adecuado ${ }^{7}$. Los cuidados del pie deberían ser abordados en forma interdisciplinaria, requiriéndose la participación de médicos, enfermeros y podólogos entrenados. La presencia de estos últimos mostró ser favorable en la prevención primaria y secundaria de estas lesiones ${ }^{8}$.

Durante las consultas, el examen rutinario del pie debería ocupar un rol protagónico. Es vital que se discrimine a los pacientes con "pie de riesgo" (ver Tabla 1) y se realice un seguimiento directo y continuo del pie que ya ha sufrido una úlcera.

Tabla 1: factores de riesgo para el desarrollo de ulceras del pie en pacientes con diabetes.

\begin{tabular}{l|l}
\cline { 2 - 2 } \multicolumn{1}{c|}{ Factor de riesgo } & \multicolumn{1}{c}{ Caracteristicas / descripción } \\
\hline Inadecuado control metabólico & Hiperglucemia, dislipemia. \\
\hline Diabetes de larga evolución & Mayor a diez años. \\
\hline Neuropata diabética & Dolor, parestesias, ins ensibilidad, quemazón, entumecimiento, alteración de la prueba del monofilamento. \\
\hline Macroangiopata diabética & $\begin{array}{l}\text { Claudicación intermitente, doior de reposo, disminución de los pulsos periféricos, palidez o cianosis del pie, blanqueo del pie a la } \\
\text { elevación del miembro, disminución de la temperatura y del vello de la pierna. }\end{array}$ \\
\hline Alteraciones de piel y faneras & Uñas encarnadas, tiña crónica del pie, onicomicosis, dishidrosis, callosidades, hemorragias en las callosidades. \\
\hline $\begin{array}{l}\text { Alteraciones biomecánicas } \\
\text { (puntos anómalos de presión) }\end{array}$ & Hallux valgus, artritis, pie cavo, pie plano, dedos en martillo o en garra. \\
\hline Hábitos nocivos coexistentes & Higiene deficiente del pie, tabaquismo, alcoholismo, uso de calzado inadecuado. \\
\hline Daño renal & Insufiencia renal, microalbuminuria. \\
\hline $\begin{array}{l}\text { Dificultades para la correcta } \\
\text { visualización del pie }\end{array}$ & Disminución de la agudeza visual, problemas de movilidad en la articulación de las rodillas o caderas. \\
\hline Otros & Situación socioeconómica desfavorable, vivir sólo, edad avanzada, actitudes psicológicas negativas, aislamiento social. \\
\hline
\end{tabular}

Modificado de: Tizòn Bouza E, et al. Atenciòn de enfermerìa en la prevenciòn y cuidados del pie diabètico. Aten Primaria 2004;34(5):263-71.

\section{Ulceras}

Manifestaciones clínicas y clasificación de las úlceras Poder identificar el origen de la úlcera al momento del examen, es de suma importancia para manejarla apropiadamente. Si bien no existen úlceras neuropáticas o vasculares puras (más bien combinaciones de ambas) las mismas podrán manifestarse con predominio de uno $\mathrm{u}$ otro origen ${ }^{4}$ :

Las úlceras de origen neuropático suelen alojarse en la cabeza de los metatarsianos y presentar un lecho rojo fácilmente sangrante, con piel circundante caliente e hiperque-ratósica y pul- sos distales respetados. En el otro extremo, las ulceras con origen predominantemente vascular suelen aparecer en zonas de traumatismo del calzado (extremo de los dedos, costados laterales de los mismos o metatarsianos) ser dolorosas, presentar un lecho atónico y fibroso, con piel circundante fría, sin hiperqueratosis, y pulsos distales no palpables².

Con el objetivo de valorar la severidad de las lesiones se han publicado diferentes clasificaciones de las úlceras de pie diabético. Las más difundidas son la de Wagner y la de la Universidad de Texas (ver Tabla 2: clasificación en grados de las úlceras del pie en pacientes con diabetes según Wagner.).

Tabla 2: clasificación en grados de las úlceras del pie en pacientes con diabetes según Wagner

\begin{tabular}{|c|c|c|c|}
\hline & & Lesión & Garacteristicas \\
\hline 0 & Ninguna & Pie de riesgo & Callos gruesos, cabezas metatarsianas prominentes, dedos en garra, deformidades ós eas. \\
\hline 1 & \multirow[t]{3}{*}{ Ulcera } & Superficial & Destrucción total del espesor de la piel. \\
\hline 2 & & Profunda $\sin$ abseso ni celulitis & Penetran en el tejido celular subcutáneo, ligamentos y músculo, pero sin afectar hueso. \\
\hline 3 & & Profunda con absceso o celulitis & Herida profunda con secreción y mal olor. Generalmente coexiste infección ósea. \\
\hline 4 & \multirow[t]{2}{*}{ Gangrena } & Limitada & Necrosis de parte del pie. \\
\hline 5 & & Extensa & Todo el pie afectado, efectos sistémicos. \\
\hline
\end{tabular}

Modificado de: Wagner FW Jr. The diabetic foot. Orthopedics 1987; 10:163-72. 
Tabla 3: clasificación en grados de las úlceras del pie en pacientes con diabetes según la Universidad de Texas.

\begin{tabular}{l|l|c|c|c}
\hline \multicolumn{1}{|c|}{$\mathbf{0}$} & $\mathbf{1}$ & $\mathbf{2}$ & $\mathbf{3}$ \\
\hline $\mathbf{A}$ & $\begin{array}{l}\text { Lesión pre-ulcerosa 0 } \\
\text { post-ulcerosa } \\
\text { completamente epitelizada }\end{array}$ & Superficial & $\begin{array}{c}\text { Ulcera } \\
\text { Que penetra en el } \\
\text { tendón o la cápsula }\end{array}$ & $\begin{array}{c}\text { Que afecta el hueso } \\
\text { y/o la articulación }\end{array}$ \\
\hline B & Más infección \\
\hline C & Más isquemia \\
\hline D & Más infección e isquemia \\
\hline
\end{tabular}

Modificado de: Levin and O'neal's. The Diabetic Foot. Sixth edition Bouker JH, Pfeifer MA editores. Mosby; 2001.

\section{Tratamiento de las úlceras del pie en pacientes con diabetes}

El tratamiento de las úlceras del pie diabético requiere de personal (enfermeros o médicos) entrenado . La presente sección pretende servir de guía para delinear los principios y el manejo básico del tratamiento de estas lesiones, con el objetivo de ayudar al profesional tratante a interactuar con el personal directamente encargado de realizar las curaciones, y para asesorar al paciente y a sus familiares sobre la evolución de las mismas. Para una reseña más detallada, se sugiere consultar la bibliografía adjunta ${ }^{11,12}$

La gran mayoría de las úlceras del pie diabético se pueden manejar en forma ambulatoria, especialmente las grado 1 y 2 de la clasificación de Wagner. Los estadios más avanzados suelen requerir inicialmente internación, usualmente para antibioticoterapia endovenosa inicial y eventual debridamiento quirúgico ${ }^{13}$.

En líneas generales, el proceso de curación implica las siguientes fases: limpieza y debridamiento, granulación y repitelización. En cada una de ellas debe prestarse, además, atención a la prevención y el tratamiento de infecciones agregadas.

Así como ocurre con el tratamiento de otros tipos de lesiones ulcerosas (por ejemplo, úlceras por decúbito, úlceras varicosas) el principio actual que rige el manejo de las úlceras del pié diabético se denomína "cura avanzada" o "cura en ambiente húmedo". Se entiende por esta a "la curación no tradicional que se efectúa limpiando la herida con suero fisiológico y dejando como cobertura un apósito interactivo, bioactivo o mixto"11. Esta metodología, a diferencia de la "cura tradicional", aplica interfases especializadas que logran mantener un ambiente con condiciones de humedad, temperatura y oxigenación más cercanas a lo fisiológico para lograr la óptima remoción de tejido desvitalizado y la posterior cicatrización de las lesiones.

En cada curación de la úlcera, el primer paso es la limpieza de la misma. El lavado de la herida se puede llevar a cabo con agua corriente templada, solución fisiológica o solución de Ringer; debiendo evitarse los antisépticos, ya que maceran la piel, son citotóxicos e inhiben la granulación ${ }^{11,12}$.

A continuación, es importante reconocer aspectos de la lesión que condicionan la estrategia de curación: la extensión y profundidad de la lesión, la presencia y la cantidad de exudado, la presencia de tejido necrótico o desvitalizado, el estado de vitalidad del lecho subyacente, la presencia o no de tejido de granulación, los signos de infección coexistentes, y el estado de la piel sana circundante a la úlcera. De acuerdo a estas variables se decidirá la modalidad de debridamiento más apropiada, se seleccionara un apósito primario -el que contiene el principio bioactivo o interactivo esencial- y eventualmente un apósito secundario -que brinda un medio de soporte adicional, cuando el apósito primario no lo provee- y se adecuará la frecuencia de reevaluación y de curaciones subsiguientes.

Existe una gran variedad de apósitos para la cura avanzada de lesiones ulcerosas. De acuerdo a su formulación, éstos pueden participar absorbiendo el exceso de secreciones del lecho, neutralizando malos olores, produciendo la lisis de detritus y tejido desvitalizado, disminuyendo la carga bacteriana y estimulando la granulación del tejido ${ }^{13}$. La utilización de antibióticos locales es controvertida, pero a la luz de las evidencias actuales se desaconseja su uso ${ }^{14,15}$.

En todos los casos es imprescindible lograr el alivio de la presión plantar ejercida por el peso corporal, pudiéndose lograr con reposo en cama -que evitará apoyar el pie-, bastones, sillas de ruedas, férulas, etc ${ }^{13}$

La tabla 4 resume los recursos terapéuticos para el tratamiento de las úlceras del pie en pacientes con diabetes.

Tabla 4: recursos terapéuticos para el tratamiento de úlceras del pie en pacientes con diabetes según su fase de curación.

\begin{tabular}{|c|c|c|c|c|}
\hline Etapa & Objetivo & Modalidiad & Alternativas & Desoripoión y Garacleristícas \\
\hline \multirow{5}{*}{ 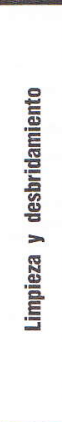 } & \multirow{5}{*}{$\begin{array}{l}\text { Remoción del tejido } \\
\text { necrótico con la } \\
\text { finalidad de obtener un } \\
\text { tejido limpio que } \\
\text { permita la cicatrización }\end{array}$} & Químico & Agua oxigenada al $5 \%$, azúcar, dextranómero & Propios de la "cura tradicional", ahora desplazados por nuevos apósitos. \\
\hline & & Enzimático & Clagenasa, fibrinolisina, hialuronidasa & $\begin{array}{l}\text { Inducen hidrólisis del tejido necrótico. Dado su escaso poder de penetración, } \\
\text { suelen utilizarse sobre ulceras superficiales. Su acción es lenta }\end{array}$ \\
\hline & & Mecánico & Arrastre con gasas & $\begin{array}{l}\text { Util para tratar lesiones grandes y profundas. La gasa actúa como agente } \\
\text { debridante primario. }\end{array}$ \\
\hline & & Quirurgico & $\begin{array}{l}\text { Bisturí, tijeras, cepillado, curetaje e } \\
\text { hidrolavado }\end{array}$ & $\begin{array}{l}\text { Util cuando hay que remover gran cantidad de tejido necrótico, sobre todo en } \\
\text { úlceras avanzadas. Suele ser doloroso y se realiza en quirófano. }\end{array}$ \\
\hline & & Autolítico & $\begin{array}{l}\text { Hidrogeles, hidrofibras, hidrocoloides, } \\
\text { cadexómero iodado y alginatos } \\
\text { poliuretanos }\end{array}$ & $\begin{array}{l}\text { Cualquier apósito capaz de crear un ambiente húmedo favorece la autodigestión } \\
\text { de los detritus por los propios macrófagos, neutrófilos y enzimas presentes en } \\
\text { los fluidos. Es el método menos traumático, más indoloro y selectivo (no afecta } \\
\text { tejidos sanos). }\end{array}$ \\
\hline 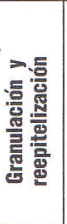 & $\begin{array}{l}\text { Estimular la síntesis de } \\
\text { colágeno y la neo } \\
\text { vascularización con el } \\
\text { posterior cierre } \\
\text { completo de las } \\
\text { heridas. }\end{array}$ & $\begin{array}{l}\text { Membranas } 0 \\
\text { apósitos } \\
\text { activos }\end{array}$ & Poliuretanos, hidrocoloides e hidrogeles & \\
\hline
\end{tabular}

Modificado y resumido de: Bileviche E. Forum de Flebologia y linfologia, 9(1): 30-36, 2007; Aburto I, Morgado P. Curación avanzada de las úlceras del pie diabético. Morgado P. editora. Publicación en linea. Disponible en: http://www.colegiodeenfermeras.cl/datos/ftp/Guia_clinica_Curacion_Pie_Diabetico.pdf. (ultimo acceso 15/06/2008); y Escuela Andaluza de Salud Pública. Tratamiento local de las úlceras por presión. En: Boletín Terapéutico Andaluz [en línea], 2000;16(5). Disponible en: http://www.easp.es/web/documentos/BTA/00001271documento.pdf. (ultimo acceso 15/06/2008). 


\section{Conclusión}

Por los diversos factores que intervienen en su desarrollo, el tratamiento de los problemas del pie en los pacientes con diabetes es complejo, de ahí la necesidad de una atención multidisciplinaria para lograr el objetivo de su curación y prevención de la recidiva de nuevas lesiones.

Las decisiones terapéuticas se basan generalmente en opinión de expertos, ya que hasta al momento no se cuenta con evi- dencia clínica suficiente como para estandarizar los tratamientos. Esto es debido a la falta de homogeneidad de los ensayos clínicos realizados hasta el momento.

Los problemas del pie en las personas con diabetes constituyen entidades prevenibles, siendo crucial para su evolución, la realización de un diagnóstico precoz. A tal efecto la educación diabetológica y la atención multidisciplinaria deberían constituirse en los pilares básicos del abordaje y la prevención de estos problemas.

\section{Citas bibliográficas}

1. Sociedad española de quimioterapia. Documento de consenso sobre el tratamiento antimicrobiano de las infecciones en el pie del diabético. Fev Esp Quimioterap 2007;20(1):77-92.

2. Viadè J. Pie Diabético. Guia práctica para la prevención evaluación y tratamiento. Madrid, España. Editorial Médica Panamericana SA, 2006.

3. Ismail K, Winkley K, Stahl D et al. A cohort study of people with diabetes and their first foot ulcer: the role of depression on mortality. Diabetes Care 2007;30(6):1473-1479.

4. Nalini S, Armstrong D, Lipsky B. Preventing Foot Ulicers in Patients with Diabetes. JAMA 2005;293:217-228

5. Rodriguez-Piñero M. Epidemiologia, repercusión sociosanitaria y etiopatogenia de las úlceras vasculares. Angiología 2003;55:260-267.

6. Van Schie C H M, Vermigli C, Carrington A L et al. Muscle Weakness and Foot Deformities in Diabetes. Diabetes Care 2004;27:1668-1673.

8. Plank J. Haas W, Rakovac I, et al. Evaluation of the Impact of Chiropodist Care in the Secondary Prevention of Foot Ulcerations in Diabetic Subjects. Diabetes Care 2003:26:1691-1695.

9. Wagner FW Jr. The diabetic foot. Orthopedics 1987;10:163-72

10. Levin and O'neal's. The Diabetic Foot. Sixth edition. Bouker JH, Pfeifer MA editors. Mosby: 2001

11. Aburto I, Morgado P. Curación avanzada de las úlceras del pie diabético. Morgado P. editora. Publicación en linea. Disponible en: hittp://www.colegiodeenfermeras.cl/datos/tip/Guia_clinica_Curacion Pie_Diabetico.pdi.

11. Aburto I, Morgado P. Curación avanzada de las úlceras del pie diabético. Morgado P. editora. Publicación en linea. Disponible en: http://www.colegiodeenfermeras.cl/datos/tip/Guia_clinica_Curacion_Pie_Diabetico.pdf.
Ultimo acceso $15 / 06 / 2008$

2. Escuela Andaluza de Salud Pública. Tratamiento local de las úlceras por presión. En: Boletín Terapéutico Andaluz [en linea], 2000;16(5). Disponible en: http://www.easp.es/web/documentos/BTA/00001271documento.pdf

Ultimo acceso 15/06/2008

13. Frykberg RG. Diabetic Foot Ulcers: Patogénesis and management. American Family Physician 2002;66(9):1955-62. Disponible en: http://www.aatp.org/atp/20021101/1655.pdf. Ultimo acceso 15/06/2008

15. Royal College of Nursing. Management of venous leg ulcer. Clinical Practice Guidelines. $1998 \mathrm{http}: / / \mathrm{www} . r \mathrm{rcn}$. org.uk/senvices/promote/clinical VenousLegUlcers.pd

\section{INFO - EVIDENCIA}

¿Qué es Evidencia, Actualización en la Práctica Ambulatoria?

Una publicación independiente editada cada dos meses desde 1997 por la Fundación MF, organización sin fines de lucro dedicada a promover el desarrollo de la medicina familiar y la atención primaria de la salud.

¿Cuál es el objetivo de Evidencia?

Contribuir a la educación continua y a la actualización de los profesionales de la salud de la región en el área de la atención ambulatoria.

\section{Contenidos de Evidencia}

- Información independiente, actualizada y resumida en forma sencilla y en castellano sobre temas relevantes de la atención ambulatoria.

- Artículos seleccionados por su calidad y relevancia clínica, resumidos y comentados críticamente por destacados profesionales del área.

- Revisiones sobre temas clínicos, epidemiológicos, sanitarios o humanísticos, de importancia para la práctica ambulatoria.

- Notas farmacológicas para la actualización rápida de temas de terapéutica clínica.

- Discusión de casos clínicos con herramientas de Medicina Basada en la Evidencia.

- Glosario de términos de epidemiología y medicina basada en la evidencia

¿Cómo es posible acceder a Evidencia?

Por suscripción a la edición en papel, o bien por Internet, en nuestro sitio: http://www.evidencia.org 